\title{
Commentary \\ Killing the umpire: cooperative defects in mitotic checkpoint and BRCA2 genes on the road to transformation
}

Frank McKeon

\author{
Harvard Medical School, Boston, Massachusetts, USA
}

Received: 25 August 1999

Accepted: 3 September 1999

Published: 7 October 1999

(c) Current Science Ltd

\begin{abstract}
Important note about how to cite this article
This article is also available online in the Breast Cancer Research website. To avoid confusion, please ensure that only the online version of the article is cited in any reference, as follows:

McKeon F: Killing the umpire: cooperative defects in mitotic checkpoint and BRCA2 genes on the road to transformation [commentary]. http://breast-cancer-research.com/vol1 no1/07oct99/ dispatch/3
\end{abstract}

\begin{abstract}
Recent findings from mouse models of BRCA2 genetic lesions have provided intriguing insights and important questions concerning modes of tumor development in familial breast and ovarian cancers. Fibroblasts from mice homozygous for the BRCA2Tr allele grow poorly and display an array of chromosomal abnormalities that are consistent with a role for $B R C A 2$ in DNA repair. This growth defect can be overcome and cellular transformation promoted by the expression of defective, dominant negative alleles of p53 and of the mitotic checkpoint gene Bub1, both of which are known to induce chromosome instability. These findings are mirrored in the genetic lesions sustained in tumors found in the rare $B R C A 2^{T r} / T r$ mice that survive to adulthood, which include defects in p53 as well as the mitotic checkpoint proteins Bub1 and Mad3L. Together, these data hint that tumors in these mice evolve from an unusually intense selective pressure to remove DNA damage checkpoints, which in turn might be facilitated by chromosomal abolition of mitotic checkpoints and the consequent increase in shuffling of genetic information. How these genetic lesions co-operate to yield transformed cells and how these data relate to BRCA1 and $B R C A 2$ defects in the human population are important questions raised by this work.
\end{abstract}

Keywords: BRCA2, Bub1, DNA repair, Mad3, mitotic checkpoint

\section{Introduction}

The BRCA1 and BRCA2 genes have attracted intense interest because their mutations have been tightly linked to inherited forms of breast and ovarian cancers, which comprise fully $20 \%$ of all cases of these malignancies [1]. Linkage analysis and positional cloning of the BRCA genes from affected families provided a rare glimpse into proteins whose malfunction leads to particular cancers and myriad biologic questions concerning underlying mechanisms. Understanding how these proteins function in the cell and why mutations predispose individuals to an often early onset of breast and ovarian cancers are considered essential milestones in efforts to mitigate these diseases.

Both BRCA1, located at $17 \mathrm{q} 21$, and BRCA2, located at 13q12-13, encode large proteins (1863 and 3418 amino acids, respectively), and are often colocalized during embryonic development to proliferating cell populations undergoing differentiation. This observation, together with molecular analyses of interaction proteins, suggest that BRCA1 and BRCA2 act in a complex with other cellular proteins, including the DNA repair protein Rad51, to monitor, signal, and correct genetic damage [2].

Murine models for BRCA1 and BRCA2 mutations have been stifled by the lack of phenotypes in the heterozygotes, and the early embryonic lethality of mice lacking both copies of BRCA1 or BRCA2 [3,4]. Considering the large number of DNA repair events required for genome duplication during a normal cell cycle, it may not be surprising that the loss of either of these genes would disrupt normal development. Significantly, both p53 and p21 are 
highly expressed in BRCA1- and BRCA2-deficient embryos, suggesting that these DNA damage checkpoint responses are intact and probably contribute to the arrested development phenotype of these mutant embryos. Indeed, the coincident loss of p53 permits somewhat extended progression of BRCA1-- and BRCA2-embryos, suggesting that p53, as well as other checkpoint proteins, are activated in these mice to induce cell cycle arrest to allow for repair processes [4].

In an effort to generate viable mouse models for $B R A C 2$ defects, more discrete lesions in the BRCA2 gene were produced that result in C-terminal truncations of the protein that corresponds to certain human germline $B R C A 2$ mutations [5]. Interestingly, some of these BRCA2TrTTr mice survive embryonic development to reach adulthood, albeit with numerous anomalies in somatic growth, poor differentiation of selected tissues, and eventually thymic lymphomas. As with the more complete loss-of-function $B R C A 2$ mutants, fibroblasts from the BRCA2Tr/Tr embryos show poor growth kinetics and high levels of p53 and p21. In addition, these fibroblasts grow more slowly with successive passages and accumulate increasing numbers of chromosomal abnormalities [5]. Thus, it is likely that the unrepaired DNA damage in the BRCA2Tr/Tr cells triggers checkpoints that in turn hinder cell proliferation.

It was this seeming paradox - a mutant tumor suppressor causing cell cycle arrest rather than permitting unbridled proliferation - which held the attention of Venkitaraman and coworkers [6], who set out to reconcile these data with the known association of BRCA2 mutations in early onset breast and ovarian cancers [1]. The result of this work was the intriguing discovery that the growth defects of cells harboring the BRCA2 $2^{\operatorname{Tr} / \mathrm{Tr}}$ alleles could be overcome in a manner to promote transformation by the loss of p53 or the mitotic checkpoint gene Bub1. Defects in these genes either directly relieve the checkpoints that monitor the abnormal BRCA2 $2^{\operatorname{r} / \mathrm{Tr}}$ chromosome structure or accelerate chromosomal aneuploidy leading to loss or gain of genetic elements that co-operate with BRCA2 defects in transformation.

\section{Mutant p53 and dominant-negative Bub1: acting in same pathway?}

Probably no image is more telling of the defect in the $B R C A 2^{T r} T_{r}$ cell lines than the mitotic chromosome spreads showing multiple breaks and alterations, presumably due to repair defects and mitotic recombination events [6]. Such events are likely to trigger the observed elevations in p53, and may also promote mitotic checkpoint genes, which ensure proper chromosome alignment and segregation.

Lee et al [6] tested this notion by expressing dominant-negative versions of $\mathrm{p} 53$ and Bub1 (N-Bub1) in the BRCA2Tr/Tr fibroblasts. Significantly, two mutant p53 alleles and a dominant-negative Bub1 construct rescued the proliferation defect in the BRCA2 $2^{\mathrm{Tr} / \mathrm{Tr}}$ cells, presumably by suppressing various checkpoints that monitor abnormal chromosome structure or segregation. The authors therefore expected that these growth-rescued cells would now be unleashed to allow even more aberrantly structured chromosomes. The N-Bub1 and the p53R273L expressing cells, although aneuploid, were surprisingly devoid of abnormal chromosomes. This was not the case for cells that expressed the other p53 allele, G154V, which also rescued the growth defect, but cells retained the abnormal chromosome structures. These intriguing results indicate several mechanisms by which mutations in checkpoint genes could cooperate with BRCA2 mutations to promote aggressive cell growth.

The continued growth of BRCA2Tr/Tr cells that express the p53 G154V mutant may be more easy to grasp as p53 acts to sense DNA damage and monitor repair, and its absence via mutation or suppression is common to many transformed cells. The apparent lack of chromosomal abnormalities in the cells expressing p53R273L or N-Bub1 is more difficult to comprehend. It is known, for instance, in the absence of p53 or by suppressing Bub1 activity via dominant-negative Bub1, cells avoid cell cycle arrest and apoptosis normally associated with the absence of chromosome alignment in mitosis [7,8]. Whereas these actions might explain the rescue of the growth inhibition of the BRCA2 $2^{T r T r}$ cells by p53R273L or N-Bub1, they fail to illuminate why the rescued cells lack chromosomal abnormalities.

Several possible mechanisms are certainly under consideration by researchers. One would have to posit that the abnormal chromosome structures observed in the BRCA2Tr/Tr cells are in fact reflections of failed repair processes, and that the assembly of the repair complexes onto chromosomes is somehow dependent on particular cell cycle arrest states mediated by p53 or components of the mitotic checkpoint. It would follow that these 'rescued' cells have as much DNA damage but appear normal due to the lack of repair intermediates. The second possibility is that the p53R273L and NBub1 mutants allow cells to bypass critical apoptotic events and therefore create a large population of cells from which those with the least chromosomal abnormalities are selected.

Although undoubtedly an area of intense research interest, this conundrum led Lee et al [6] to examine the thymic lymphomas arising in the BRCA2 $2^{T r / T r}$ mice for chromosomal abnormalities. Remarkably, cells from these tumors were free of significant chromosomal defects, suggesting a similar loss in checkpoint activities as seen in the $B R C A 2^{T r} / T r$ fibroblasts that express p53R273L or N-Bub1. Analysis of the small sample of tumors available (only 1 in 100 of the BRCA2Tr/Tr mice survive to adulthood) revealed that all had defects in $p 53, B u b 1$, or the Bub1-related gene Mad3L. Curiously, three of the lymphomas showed a similar spectrum of genetic defects involving unusual heterozygous deletions of a region around amino acids 
$140-148$ of the p53 gene, as well as identical, in-frame deletion/insertions in the Bub1 gene substituting M290 with codons encoding isoleucine and arginine. A fourth lymphoma showed a heterozygous mutation in the Mad3L gene and lacked obvious changes in $p 53$ or Bub1. Interestingly, all of the p53, Bub1, and Mad3L mutants functioned as dominant-negative agents in cells with regard to the mitotic checkpoint, suggesting that the lymphoma cells are defective in these functions despite the presence of a single wild-type gene.

The work of Lee et al [6] has implications for mechanisms of cell transformation in general and tumorigenesis involving BRCA2 mutations in particular. For one, it is the second indication, following that of Cahill et al [9], that mutations in mitotic checkpoint regulators participate in the genomic instability thought to promote tumorigenesis. In the latter case, mutations in Bub1 and Mad3L (BubR1) were found in colorectal carcinoma cell lines displaying a particular genomic instability marked by rapid loss and gains of entire chromosomes (chromosome instability) [9]. It should be noted here that analyses of the Bub1 gene in head and neck squamous cell carcinomas, typically aneuploid tumors, failed to detect mutations [10]. Moreover, Cahill et al [9] found that only several of the colorectal carcinoma cell lines displaying the chromosome instability phenotype showed mutations in the Bub1 or Mad3L genes. It is likely that many, yet to be discovered genes play critical roles in the various checkpoint pathways that govern accurate chromosome segregation, and mutations in such genes could contribute to the genetic instability that promotes tumor cell evolution.

\section{Conclusion}

With regard to tumor development in cells that harbor BRCA2 mutations, the findings of Lee et al [6] are sure to stimulate much discussion and many new experiments. The data are increasing that breast and ovarian tumors that arise in individuals who harbor BRCA1 or BRCA2 mutations show frequent and unusual p53 genetic defects, suggesting particular pathways of tumor development in these patients $[11,12]$. In this regard, the intriguing models presented by Lee $e t$ al indicate that cells homozygous for the BRCA2 2 Tr allele almost require checkpoint defects in order to merely proliferate, therefore providing additional selective pressure to remove checkpoint mechanisms by genetic lesions. Given that such alterations in p53, together with those in genes that are thought to specifically govern mitotic chromosome alignment, rescue the growth defects in BRCA2Tr/Tr cells, the end product has both poor DNA repair capabilities but no failsafe mechanisms to deter their continued propagation. These cells might therefore be expected to evolve rapidly through chromosome aneuploidy and an unbridled accumulation of mutations, which together would yield a dangerous brew of progeny upon which growth selection operates.
The issue of cooperating mutations coinciding with BRCA1 and BRCA2 lesions brings forth another interesting observation from the paper by Lee et al [6] concerning p53 and mitotic checkpoint mutations. Although not tested directly, evidence is mounting that $\mathrm{p} 53$, in addition to its other well established functions, mediates the cell cycle arrest and apoptosis that follows the activation of the mitotic checkpoint. It is therefore curious that most of the thymic lymphomas of the BRCA2Tr/Tr mice showed mutations both in p53 and Bub1, suggesting some additional advantage for the loss of both activities. Obviously this result suggests a more complex story than Bub1 activating p53 to guard against aneuploidy, but then again the intricacies of mechanisms that ensure chromosome segregation have only recently been addressed. Whereas p53 mutations are more frequent in $B R C A 1$ and $B R C A 2$ tumors than in sporadic cases of breast cancer, it will of significant interest to determine whether mutations in mitotic checkpoint proteins, including Bub1, Bub3, Mad1, Mad2, and Mad3L, contribute to the progression of human BRCA1 and BRCA2 tumors [13]. It is certain that such analyses will yield additional surprises that impact both on the biology of mitosis and on the pathology of cancer cells.

\section{References}

1. Ellisen LW, Haber DA: Hereditary breast cancer. Annu Rev Med 1998, 49:425-436.

2. Irminger-Finger I, Siegel BD, Leung WC: The functions of breast cancer susceptibility gene 1 (BRCA1) product and its associated proteins. Biol Chem 1999, 380:117-128.

3. Ghebranious N, Donehower LA: Mouse models in tumor suppression. Oncogene 1998, 17:3385-3400.

4. Ludwig T, Chapman DL, Papaioannou VE, Efstratiadis A: Targeted mutations of breast cancer susceptibility gene homologs in mice: lethal phenotypes of Brca1, Brca2, Brca1/Brca2, Brca1/p53, and Brca2/p53 nullizygous embryos. Genes Dev 1997, 11:1226-1241.

5. Patel KJ, Vu VP, Lee H, et al: Involvement of Brca2 in DNA repair. Mol Cell 1998, 1:347-357.

6. Lee $\mathrm{H}$, Trainer $\mathrm{AH}$, Friedman LS, et al: Mitotic checkpoint inactivation fosters transformation in cells lacking the breast cancer susceptibility gene, Brca2. Mol Cell 1999, 4:1-10.

7. Taylor SS, McKeon F: Kinetochore localization of murine Bub1 is required for normal mitotic timing and checkpoint response to spindle damage. Cell 1997, 89:727-735.

8. Lengauer C, Kinzler KW, Vogelstein B: Genetic instabilities in human cancers. Nature 1998, 396:643-649.

9. Cahill DP, Lengauer C, Yu J, et al: Mutations of mitotic checkpoint genes in human cancers. Nature 1998, 392:300-303.

10. Yamaguchi K, Okami K, Hibi K, et al: Mutation analysis of hBUB1 in aneuploid HNSCC and lung cancer cell lines. Cancer Lett 1999, 139:183-187.

11. Ramus SJ, Bobrow LG, Pharoah PD, et al: Increased frequency of TP53 mutations in BRCA1 and BRCA2 ovarian tumours. Genes Chromosomes Cancer 1999, 25:91-96.

12. Smith PD, Crossland S, Parker G, et al: Novel p53 mutants selected in BRCA-associated tumours which dissociate transformation suppression from other wild-type p53 functions. Oncogene 1999, 18:2451-2459.

13. Amon A: The spindle checkpoint. Curr Opin Genet Dev 1999, 9:69-75.

Author address: Department of Cell Biology, Harvard Medical School, Boston, Massachusetts, USA

Correspondence: Frank McKeon, Department of Cell Biology, Harvard Medical School, 240 Longwood Avenue, Boston, MA 02115, USA.

Tel: 617432 0994; fax: 617432 6655;

e-mail: frank_mckeon@hms.harvard.edu 